OPEN ACCESS

Edited by:

Gary Iwamoto,

University of Illinois at

Urbana-Champaign, United States

Reviewed by:

Bryan Blissmer,

University of Rhode Island,

United States

*Correspondence:

Yi-Yuan Tang

yiyuan.tang@ttu.edu

Marjorie Woollacott

mwool@uoregon.edu

Specialty section

This article was submitted to

Exercise Physiology,

a section of the journal

Frontiers in Physiology

Received: 09 July 2020 Accepted: 09 September 2020

Published: 23 October 2020

Citation:

Tang Y-Y and Woollacott M (2020)

Editorial: The Physiological and

Genetic Influences of Meditation and

Tai Chi on Mental, Emotional, and

Movement Regulation.

Front. Physiol. 11:581841.

doi: 10.3389/fphys.2020.581841

\section{Editorial: The Physiological and Genetic Influences of Meditation and Tai Chi on Mental, Emotional, and Movement Regulation}

\author{
Yi-Yuan Tang $^{1 *}$ and Marjorie Woollacott ${ }^{2 *}$ \\ ${ }^{1}$ Department of Psychological Sciences, Texas Tech University, Lubbock, TX, United States, ${ }^{2}$ Department of Human \\ Physiology, University of Oregon, Eugene, OR, United States
}

Keywords: meditation, Tai Chi, physiology, genetic influences, brain, self-regulation, bodifulness, mindfulness

\section{Editorial on the Research Topic}

The Physiological and Genetic Influences of Meditation and Tai Chi on Mental, Emotional, and Movement Regulation

Growing evidence shows the efficacy of mind-body interventions such as meditation and Tai Chi and suggested underlying mechanisms (Gatts and Woollacott, 2007; Tang et al., 2015; Wu et al., 2018; Liu et al., 2019; Cheng et al., 2020). However, little is known about their physiological and genetic influences on mental, emotional, and movement regulation.

Tai Chi is a slow, gentle mind-body practice facilitating sensory awareness of the speed, force, and execution of movement. In practice, the body is naturally relaxed and extended, the mind is calm but alert, and body postures and movements are coordinated. Tai Chi practice involves balanced postures that flow from one to another and promote concentration and mindfulness to improve motor efficiency, flexibility, and optimize motor control (Li et al., 2001). These components can be termed bodifulness and mindfulness (Tang et al., 2015, 2019).

In this Research Topic, we collected recent findings using unique and well-validated measures in healthy and patient populations. Using a classical reach-to-grasp task, Sartori et al. showed that a Tai Chi group improved motor efficiency and flexibility compared to untrained controls. Mild cognitive impairment (MCI) reduces finger tapping performance (FTP), an index of motor function. Zhang et al. used infrared photoelectric sensing to detect FTP in elderly groups-healthy vs. MCI subjects with/out Tai Chi practice. Results showed that FTP in both hands in MCI subjects was lower than in healthy subjects. However, in the Tai Chi group, only the dominant right hand's FTP of MCI subjects was lower than in healthy subjects; left hand's FTP remained normal, suggesting Tai Chi could increase motor function in MCI subjects. Major depressive disorder (MDD) accompanies depressed mood and low vitality/fatigue. Xu et al. showed that Tai Chi improved mood and vitality in MDD, accompanying insular functional connectivity changes via fMRI related to mood/fatigue improvements. Tai Chi also has protective effects on coronary heart disease (CHD). At the molecular level, serum miR-24 and miR-155 levels relate to CHD severity. Li et al. reported that Tai Chi improved CHD prognosis, affecting serum levels of miR-24 and miR-155. 
Mindfulness meditation (MM) is popular but its definition is in debate. Some claim that mindfulness-based stress reduction (MBSR) is an exemplar of MM that fosters mindfulness, but other variations are interventions that incorporate mindfulness as just one component (Creswell, 2017). However, this might be misleading since MBSR consists of multiple components (KabatZinn, 1990; Davidson and Kabat-Zinn, 2004; Smith, 2004; Tang et al., 2017). For example, Valim et al. compared one session of a positive emotion-based meditation focused on gratitude (EBM) and classical MM using an emotional regulation task and found that EBM outperformed MM. Notably, EBM included the practice of mindfulness and added emotional-regulation training. Therefore, the term mindfulness meditation should not define the nature of a program. Instead, the exact components and instructions of mindfulness practice are the key. For details on meditation-related outcomes, please see reviews (Chiesa and

\section{REFERENCES}

Black, D. S., and Slavich, G. M. (2016). Mindfulness meditation and the immune system: a systematic review of randomized controlled trials. Ann. N. Y. Acad. Sci. 1373, 13-24. doi: 10.1111/nyas.12998

Cheng, D., Wang, B., Li, Q., Guo, Y., and Wang, L. (2020). Research on function and mechanism of Tai Chi on cardiac rehabilitation. Chin. J. Integr. Med. 26, 393-400. doi: 10.1007/s11655-020-3262-9

Chiesa, A., and Malinowski, P. (2011). Mindfulness-based approaches: are they all the same? J. Clin. Psychol. 67, 404-424. doi: 10.1002/jclp.20776

Creswell, J. D. (2017). Mindfulness interventions. Annu. Rev. Psychol. 68, 491-516. doi: 10.1146/annurev-psych-042716-051139

Davidson, R., and Kabat-Zinn, J. (2004). Letters to the editor. Psychosom. Med. 66,149-152. doi: 10.1097/00006842-200401000-00023

Gatts, S. K., and Woollacott, M. H. (2007). How Tai Chi improves balance: biomechanics of recovery to a walking slip in impaired seniors. Gait Posture 25, 205-214. doi: 10.1016/j.gaitpost.2006.03.011

Goyal, M., Singh, S., Sibinga, E. M., Gould, N. F., Rowland-Seymour, A., Sharma, R., et al. (2014). Meditation programs for psychological stress and well-being: a systematic review and meta-analysis. JAMA Intern. Med. 174, 357-368. doi: 10.1001/jamainternmed.2013.13018

Hölzel, B. K., Lazar, S. W., Gard, T., Schuman-Olivier, Z., Vago, D. R., and Ott, U. (2011). How does mindfulness meditation work? Proposing mechanisms of action from a conceptual and neural perspective. Perspect. Psychol. Sci. 6, 537-559. doi: 10.1177/1745691611419671

Kabat-Zinn, J. (1990). Full Catastrophe Living: Using the Wisdom of Your Body and Mind to Face Stress, Pain and Illness. New York, NY: Delacorte.

Li, J. X., Hong, Y., and Chan, K. M. (2001). Tai chi: physiological characteristics and beneficial effects on health. Br. J. Sports Med. 35:148. doi: 10.1136/bjsm.35.3.148

Liu, J., Tao, J., Liu, W., Huang, J., Xue, X., Li, M., et al. (2019). Different modulation effects of Tai Chi Chuan and Baduanjin on resting-state functional connectivity
Malinowski, 2011; Hölzel et al., 2011; Goyal et al., 2014; Tang et al., 2015; Black and Slavich, 2016; Russell-Williams et al., 2018).

In summary, this Research Topic covers the main forms of mind-body practice to reveal effects at psychological, physiological, neurobiological, and molecular levels, resulting in deepening our understanding of underlying mechanisms and providing new insights for developing appropriate behavioral interventions.

\section{AUTHOR CONTRIBUTIONS}

Y-YT and MW drafted, revised, and finalized the manuscript.

\section{FUNDING}

This work was supported by NIH and ONR.

of the default mode network in older adults. Soc. Cogn. Affect. Neurosci. 14, 217-224. doi: 10.1093/scan/nsz001

Russell-Williams, J., Jaroudi, W., Perich, T., Hoscheidt, S., El Haj, M., and Moustafa, A. A. (2018). Mindfulness and meditation: treating cognitive impairment and reducing stress in dementia. Rev. Neurosci. 29, 791-804. doi: 10.1515/revneuro-2017-0066

Smith, J. C. (2004). Alterations in brain and immune function produced by mindfulness meditation: three caveats. Psychosom. Med. 66, 148-149. doi: 10.1097/00006842-200401000-00022

Tang, Y. Y., Holzel, B. K., and Posner, M. I. (2015). The neuroscience of mindfulness meditation. Nat. Rev. Neurosci. 16, 213-225. doi: 10.1038/nrn3916

Tang, Y. Y., Jiang, C., and Tang, R. (2017). How mind-body practice worksintegration or separation? Front. Psychol. 8:866. doi: 10.3389/fpsyg.2017.00866

Tang, Y. Y., Tang, R., and Gross, J. (2019). Promoting psychological well-being through an evidence-based mindfulness training program. Front. Hum. Neurosci. 13:237. doi: 10.3389/fnhum.2019.0 0237

Wu, S., Chen, J., Wang, S., Jiang, M., Wang, X., and Wen, Y. (2018). Effect of Tai Chi exercise on balance function of stroke patients: a meta-analysis. Med. Sci. Monit. Basic Res. 24, 210-215. doi: 10.12659/MSMBR.911951

Conflict of Interest: The authors declare that the research was conducted in the absence of any commercial or financial relationships that could be construed as a potential conflict of interest.

Copyright (C) 2020 Tang and Woollacott. This is an open-access article distributed under the terms of the Creative Commons Attribution License (CC BY). The use, distribution or reproduction in other forums is permitted, provided the original author(s) and the copyright owner(s) are credited and that the original publication in this journal is cited, in accordance with accepted academic practice. No use, distribution or reproduction is permitted which does not comply with these terms. 